\title{
BMJ Open Economic burden of paediatric-onset disabilities among young and middle- aged adults in the USA: a cohort study of privately insured beneficiaries
}

\author{
Daniel Whitney, ${ }^{\oplus 1,2}$ Neil Kamdar, ${ }^{2}$ Richard A Hirth, ${ }^{3}$ Edward A Hurvitz, ${ }^{1}$ \\ Mark D Peterson ${ }^{1,2}$
}

To cite: Whitney D, Kamdar $\mathrm{N}$, Hirth RA, et al. Economic burden of paediatric-onset disabilities among young and middle-aged adults in the USA: a cohort study of privately insured beneficiaries. BMJ Open 2019;9:e030490. doi:10.1136/ bmjopen-2019-030490

- Prepublication history and additional material for this paper are available online. To view these files, please visit the journal online (http://dx.doi. org/10.1136/bmjopen-2019030490).

Received 20 March 2019 Revised 23 July 2019 Accepted 24 July 2019

Check for updates

(C) Author(s) (or their employer(s)) 2019. Re-use permitted under CC BY-NC. No commercial re-use. See rights and permissions. Published by BMJ.

${ }^{1}$ Department of Physical Medicine and Rehabilitation, University of Michigan, Ann Arbor, Michigan, USA

${ }^{2}$ Institute for Healthcare Policy and Innovation, University of Michigan, Ann Arbor, Michigan, USA

${ }^{3}$ Department of Health

Management and Policy, University of Michigan, Ann Arbor, Michigan, USA

Correspondence to Dr Daniel Whitney; danielwhitney8@gmail.com

\section{ABSTRACT}

Objective Individuals with paediatric-onset disabilities (PoDs) have complex healthcare needs and are susceptible to adverse health outcomes, which may impose a higher strain on healthcare resources. The burden of healthcare resource utilisation and costs attributed to the population of adults with PoDs is not clearly established. The objective here was to compare healthcare resource utilisation and costs between adults with versus without PoDs.

Design Cohort.

Setting Data were from the 2016 Optum Clinformatics Data Mart, a de-identified nationwide claims database of beneficiaries from a single private payer in the USA. Participants International Classification of Diseases, Tenth Revision, Clinical Modification diagnosis codes were used to identify beneficiaries with PoDs that were between 18 and 64 years of age.

Primary and secondary outcome measures Annual allcause healthcare resource utilisation and total healthcare costs were compared between adults with and without PoDs before and after adjusting for sociodemographics and several costly non-communicable diseases.

Results Adults with PoDs ( $n=121446)$ had greater annual mean counts of service utilisation for all service types (eg, inpatient, outpatient, emergency visits) compared with adults without PoDs ( $n=5415$ 475) before and after adjustments (all $p<0.001$ ). Adults with PoDs had greater unadjusted total standardised reimbursement costs (US\$26702 vs US\$8464; mean difference=US\$18 238; cost ratio $(\mathrm{CR})=3.16 ; 95 \% \mathrm{Cl}=3.13$ to 3.18 ) and total patient out-of-pocket costs (US\$2226 vs US\$1157; mean difference=US $\$ 1069 ; \mathrm{CR}=1.88 ; 95 \% \mathrm{Cl}=1.86$ to 1.89 ). After adjustments, total standardised reimbursement costs were 2.32 times higher $(95 \% \mathrm{Cl}=2.30$ to 2.34$)$ and total patient out-of-pocket costs were 1.65 times higher $(95 \%$ $\mathrm{Cl}=1.64$ to 1.66 ) compared with adults without PoDs. Conclusion Adults with PoDs had greater healthcare utilisation and costs, even after accounting for costly diseases. Future research is needed to identify the cost drivers for adults with PoDs.

\section{INTRODUCTION}

Paediatric-onset disabilities (PoDs) refer to a group of congenital or acquired conditions that originate at conception, during
Strengths and limitations of this study

- This is the largest study to date to examine healthcare utilisation and associated costs of adults with different types of paediatric-onset disabilities.

- We examined the healthcare-related economic burden after accounting for costly non-communicable diseases that are more prevalent among adults with paediatric-onset disabilities.

- This study leveraged administrative claims data, and are therefore subject to limitations that are inherent to claims data, such as inaccurate coding of medical conditions.

We were unable to determine specific cost drivers.

pregnancy or in childhood, and consist of impairments in behavioural, intellectual and/or physical functioning, or are associated with abnormal developmental or metabolic processes. While clinical care and coordinated healthcare management (eg, specialist referral) for children with PoDs is a large focus of paediatric clinicians and researchers, far less attention on the topic has been given to these individuals as they become adults. The complex healthcare needs and health complications born out of direct (eg, genetic) and resulting (eg, delayed growth) consequences associated with PoDs during growth and development have lasting ramifications on the health and functional status across the lifespan. Research has shown that individuals with PoDs are susceptible to early development of chronic, non-communicable diseases and early mortality, ${ }^{1-13}$ thus placing a high strain on the patients, caregivers and healthcare resources. Importantly, the population of adults with PoDs is projected to expand over the coming decades, ${ }^{14-16}$ which may lead to a noticeable increase in the national healthcare economic burden, and should 
be considered an urgent public health issue. However, healthcare resource utilisation and costs directly attributable to adults with PoDs has not been extensively studied.

The current US healthcare system may not be adequately prepared to care for adults with PoDs. ${ }^{17-20}$ To date, little is known about the natural trajectory of adverse health complications across the lifespan for these populations, best practices for coordinating healthcare services between general physicians and specialists (eg, paediatricians) and how to provide adequate resources for preventive and treatment services to these populations. A comprehensive understanding of healthcare resource utilisation and costs among adults with PoDs is crucial for public health, healthcare policy reform and implementing better clinical practice guidelines for cost-effective care. Furthermore, understanding the healthcare economic burden among at-risk populations prior to reaching the elderly years could inform public health efforts, policymakers and early patient and clinical decision-making processes and strategies (eg, surgeries, medications) to maximise comorbidity management and prevention. Therefore, the primary aim of this study was to characterise healthcare resource utilisation and costs among young and middle-aged adults with PoDs, as compared with young and middle-aged adults without PoDs, before and after adjusting for costly comorbid non-communicable diseases. We hypothesised that adults with PoDs would have higher healthcare resource utilisation and costs compared with adults without PoDs, even after adjusting for non-communicable diseases.

\section{METHODS}

\section{Data source}

Data were from the 2016 Clinformatics Data Mart Database (OptumInsight, Eden Prairie, Minnesota, USA), which is a nationwide, de-identified single private payer administrative claims database. This database contains over 16 million beneficiaries in 2016 who have either commercial or Medicare Advantage health plans, and includes all the inpatient, outpatient, pharmacy, emergency visit, office visit and other ancillary service utilisation throughout their enrolment on the insurance plan. Medicare beneficiaries can opt to enrol in a private Medicare Advantage health plan in lieu of participating in the traditional public Medicare programme. Such plans can offer extra coverage not available in the traditional Medicare programme, such as vision, hearing, dental and/ or health and wellness programmes. To be enrolled in a private payer health plan, beneficiaries of any age, income or disability status either pay for coverage or are covered through their employer. Administrative claims data are primarily used for billing reimbursement purposes, and health conditions are identified using specific codes attached to individual claims. Since data are de-identified, the University Institutional Review Board approved this study as non-regulated.

\section{Sample selection}

Beneficiaries that were 18-64 years of age, had 12 full months of continuous enrolment and had at least one service utilisation in 2016 (to make a diagnosis of PoDs and non-communicable diseases) were considered for this investigation. Beneficiaries were excluded if they did not have known data for sex $(n=991,<0.01 \%)$. All PoDs and non-communicable diseases were identified using the International Classification of Diseases, Tenth Revision, Clinical Modification codes, and are presented in online supplementary table 1 . Since it is not possible to determine if some conditions developed in childhood or adulthood (eg, spinal cord injury, cancer), conditions were included in the group representing PoDs if there were known childhood origins. These conditions were then grouped into the following PoD subcategories: PoDs of the musculoskeletal system; PoDs of the circulatory system; neurodevelopmental PoDs; PoDs of the urinary system; PoDs of the respiratory system and digestive system; PoDs of the nervous system; PoDs of the genital organs; PoDs of malformations of the eye, ear, face and neck and other chromosomal abnormalities.

\section{Non-communicable diseases}

Prevalent non-communicable diseases, as individual dichotomous variables (present vs not present), were ascertained by at least one medical claim in 2016, and were selected with guidance from the literature on disease, disability and mortality among adults. ${ }^{2}{ }^{3}$ 21-26 Non-communicable diseases were represented as categories across multiple biological systems as follows: ischaemic heart diseases (eg, atherosclerotic heart disease); cerebrovascular diseases (eg, cerebral infarction); hypertensive and other cardiovascular diseases (eg, hypertension, heart failure); type 2 diabetes mellitus; malignant cancer; osteoporosis; mood affective disorders (eg, depression); chronic obstructive pulmonary diseases (eg, emphysema); chronic kidney diseases (eg, kidney disease stages I-V) and liver diseases (eg, cirrhosis).

\section{Healthcare resource utilisation}

Annual all-cause healthcare resource utilisation was identified using medical claims and was categorised into the following service types: inpatient; outpatient, ambulatory and other (herein referred to as 'outpatient and other'); emergency department and office. Specific services per service category are presented in table 1 . Measures to assess healthcare resource utilisation included the percentage of patients per group (with PoDs, without PoDs) that had at least one service utilisation within each service category, and the annual mean count of service utilisation per group for each service category.

\section{Healthcare costs}

Annual all-cause healthcare costs were identified using medical and outpatient pharmacy (herein referred to as 'pharmacy') claims, and were from the paid amounts of adjudicated (final action) claims, which included insurer 
Table 1 Healthcare resource utilisation by service type

\begin{tabular}{ll}
\hline Healthcare service type & Level 1 description: level $\mathbf{2}$ description \\
\hline $\begin{array}{l}\text { Inpatient services } \\
\text { Outpatient, ambulatory and } \\
\text { other services }\end{array}$ & $\begin{array}{l}\text { Facility inpatient: acute and rehab/skilled nursing facility; professional services: inpatient visits } \\
\text { professional services: allergy tests and injections, anaesthesia, consultations, diagnostic } \\
\text { testing, immunisations and injections, laboratory, mental health, obstetrics, pathology, } \\
\text { physical medicine/rehab, professional other, preventive medicine, radiology, surgery, vision, } \\
\text { hearing and speech }\end{array}$ \\
$\begin{array}{l}\text { Emergency department } \\
\text { services } \\
\text { Office services }\end{array}$ & \begin{tabular}{l} 
Facility outpatient: emergency room; professional services: emergency room \\
\hline
\end{tabular} \\
\hline
\end{tabular}

and health plan payments and patient copayments, deductibles and coinsurance. Total (medical+pharmacy), medical and pharmacy all-cause healthcare costs were represented as standardised reimbursement cost (ie, sum of all health plan and patient paid amounts) and patient out-of-pocket cost (ie, sum of copayment, deductible and coinsurance).

Clinformatics Data Mart Database accounts for differences in pricing across health plans and provider contracts by standardising cost field used algorithms that reflect the allowed payments across all provider services. This allows comparisons across patients, data sources and geographic areas, and accounts for contractual payer and provider differences. Price standardisation accounts for quantity of services provided, relative resource costs involved in providing the services and the nature of the service which can be defined as the Current Procedural Terminology codes/Healthcare Common Procedure Coding System for professional services, National Drug Codes for pharmacy service or type of admission for inpatient stay.

\section{Sociodemographic variables}

Age, sex, ethnicity, education level, household annual income and insurance coverage (ie, commercial only, Medicare Advantage) were considered for risk adjustment to differentiate the effect of potential confounders from the effect of PoDs on the outcome.

\section{Statistical analysis}

To limit extreme values and to reduce the effect of possible spurious outliers, winsorization of healthcare resource utilisation data to the upper $99.0 \%$ was performed for each service category.

Descriptive characteristics and healthcare resource utilisation and cost measures were summarised using mean \pm SD for continuous variables and percentage for categorical variables. Group differences for healthcare resource utilisation measures were examined using $\chi^{2}$ tests for unadjusted analyses of binary data (eg, sex, education level, non-communicable diseases). Generalised linear models assuming a zero-inflated Poisson distribution (for non-utilisation) and log-link function were performed for count data (ie, number of visits per service type) to estimate the ratio of means (exponentiated form of variable estimate which represents a multiplicative effect on the estimated mean count per one unit change in the explanatory variable ${ }^{27}$ for explanatory variables (eg, PoD group), while controlling for age, sex, education, insurance coverage and all non-communicable diseases. As recommended for healthcare cost data analysis, ${ }^{28} 29$ generalised linear models with gamma distribution and log-link function were performed to estimate the CRs (exponentiated form of variable estimate) for explanatory variables (eg, PoD group), while controlling for age, sex, education, insurance coverage and all non-communicable diseases. For the generalised linear models, the main effect for PoD group was interpreted. We adjusted the models for the non-communicable diseases to determine the effect of PoDs on the outcome beyond the presence of these diseases, because they can incur excess healthcare utilisation and costs which may explain any differences in costs between groups. Ethnicity and household annual income were not initially included as covariates because of the extent of unknown/missingness. However, because these variables can be associated with healthcare delivery and non-communicable diseases, we conducted a sensitivity analysis using the fully adjusted generalised linear models that included ethnicity and household annual income to determine if these variables biassed the estimated CR for group (reference: without PoDs) for healthcare cost measures.

To determine whether there is variation in the effect of different PoD categories, healthcare resource utilisation and total cost measures were summarised using mean \pm SD after stratifying the type of PoD (eg, musculoskeletal system, neurodevelopmental). Comparisons between each of the PoD categories with the sample without PoDs were examined for healthcare resource utilisation and cost measures using the same procedures and models noted above.

Finally, to determine if age was differentially associated with healthcare costs for adults with and without PoDs, we examined the age (young, 18-40years; middle-aged, 41-64years) by group interaction for all individuals with PoDs compared with the sample without PoDs, and then for each of the PoD categories compared with the sample without PoDs. The outcome measures were total 
standardised reimbursement costs and patient out-ofpocket costs, as these were the primary outcome measures of interest. If the interaction was significant, subsequent analyses were performed after stratifying by age group.

All analyses were performed using SAS V.9.4 (SAS Institute, Cary, North Carolina, USA). Effect estimates were reported as CRs with $95 \%$ CI, and $\mathrm{p} \leq 0.05$ (two-tailed) was used to determine statistical significance.

\section{Patient and public involvement}

Patient were not directly involved in the design or conduct of this study.

\section{RESULTS}

Descriptive characteristics of individuals with PoDs $(\mathrm{n}=121446)$ and without PoDs ( $\mathrm{n}=5415$ 475) are presented in table 2. Notably, individuals with PoDs had a higher proportion enrolled in the Medicare Advantage health plan compared with individuals without PoDs. Furthermore, individuals with PoDs had higher prevalence of non-communicable diseases compared with individuals without PoDs, including: ischaemic heart disease $(8.7 \%, 3.5 \%)$; cerebrovascular disease $(6.3 \%, 1.5 \%)$; hypertensive or other cardiovascular disease $(37.8 \%$, $25.4 \%)$; type 2 diabetes $(14.5 \%, 10.0 \%)$; malignant cancer $(6.6 \%, 3.8 \%)$; osteoporosis $(3.4 \%, 1.3 \%)$; mood affective disorders $(20.8 \%, 11.0 \%)$; chronic obstructive pulmonary disease $(1.8 \%, 0.7 \%)$; chronic kidney disease $(6.6 \%$, $1.8 \%)$ and liver disease $(7.3 \%, 2.9 \%)$ (all $\mathrm{p}<0.001)$.

\section{Healthcare resource utilisation for adults with and without PoDs}

Individuals with PoDs had higher prevalence of patients with at least one service used compared with individuals without PoDs for inpatient visits $(22.5 \%$ vs $7.0 \%)$, outpatient and other visits ( $98.9 \%$ vs $94.8 \%)$, emergency department visits $(33.6 \%$ vs $18.6 \%)$ and office visits $(96.4 \%$ vs $85.6 \%$ ) (all $\mathrm{p}<0.001)$. Individuals with PoDs had greater unadjusted annual mean count of service utilisation compared with individuals without PoDs (presented as mean $\pm \mathrm{SD})$ for inpatient visits $(1.0 \pm 2.4$ vs $0.2 \pm 1.2)$, outpatient and other visits $(14.7 \pm 13.4$ vs $7.1 \pm 8.9)$, emergency department visits $(0.6 \pm 1.1$ vs $0.3 \pm 0.7)$ and office visits $(7.4 \pm 5.8$ vs $3.8 \pm 4.2)($ all $\mathrm{p}<0.001)$ (data not shown). The higher service utilisation was present even after adjusting for age, sex, education, insurance coverage and all non-communicable diseases for each service category (all $\mathrm{p}<0.001$; online supplementary table 2 ).

\section{Healthcare costs for adults with and without PoDs}

Unadjusted annual all-cause healthcare costs for individuals with and without PoDs are presented in figure 1. The standardised reimbursement costs (figure 1A) were higher for individuals with PoDs compared with individuals without PoDs for total (mean difference=US $\$ 18$ 238; $\mathrm{CR}=3.16 ; 95 \% \mathrm{CI}=3.13$ to 3.18 ), medical (mean difference=US $\$ 16445 ; \mathrm{CR}=3.39 ; 95 \% \mathrm{CI}=3.36$ to 3.42 )
Table 2 Descriptive characteristics and prevalence of noncommunicable diseases among adults (aged 18-64 years) with and without PoDs

\begin{tabular}{|c|c|c|c|}
\hline & $\begin{array}{l}\text { PoD } \\
(n=121446)\end{array}$ & $\begin{array}{l}\text { Without PoD } \\
(n=5415475)\end{array}$ & \\
\hline & $\begin{array}{l}\text { Point } \\
\text { estimate }\end{array}$ & $\begin{array}{l}\text { Point } \\
\text { estimate }\end{array}$ & $P$ value \\
\hline \multicolumn{4}{|l|}{ Descriptive characteristics } \\
\hline Age, mean (SD) & $44.3(13.6)$ & $43.8(12.9)$ & $<0.001$ \\
\hline $18-40$ years, $\%$ & 38.8 & 40.3 & \\
\hline $41-64$ years, \% & 61.2 & 59.7 & \\
\hline Sex, \% & & & $<0.001$ \\
\hline Female & 55.6 & 53.8 & \\
\hline Male & 44.4 & 46.2 & \\
\hline Ethnicity, \% & & & $<0.001$ \\
\hline White & 55.7 & 53.4 & \\
\hline Black & 7.9 & 7.4 & \\
\hline Hispanic & 8.3 & 9.4 & \\
\hline Asian & 3.0 & 4.3 & \\
\hline Unknown/missing & 25.0 & 25.5 & \\
\hline Education, \% & & & $<0.001$ \\
\hline Less than high school & 0.4 & 0.5 & \\
\hline High school diploma & 25.1 & 24.0 & \\
\hline More than high school & 71.9 & 73.5 & \\
\hline Unknown/missing & 2.6 & 2.1 & \\
\hline $\begin{array}{l}\text { Household annual income } \\
\text { (US\$), \% }\end{array}$ & & & $<0.001$ \\
\hline$<40 \mathrm{~K}$ & 15.2 & 13.1 & \\
\hline $40 \mathrm{~K}-59.9 \mathrm{~K}$ & 9.9 & 10.0 & \\
\hline $60 \mathrm{~K}-99.9 \mathrm{~K}$ & 19.0 & 20.0 & \\
\hline$\geq 100 \mathrm{~K}$ & 32.6 & 34.1 & \\
\hline Unknown/missing & 23.4 & 22.8 & \\
\hline Insurance coverage, \% & & & $<0.001$ \\
\hline Commercial only & 78.9 & 93.4 & \\
\hline Medicare Advantage & 21.1 & 6.6 & \\
\hline \multicolumn{4}{|l|}{ Non-communicable diseases } \\
\hline Ischaemic heart disease, \% & 8.7 & 3.5 & $<0.001$ \\
\hline Cerebrovascular disease, $\%$ & 6.3 & 1.5 & $<0.001$ \\
\hline $\begin{array}{l}\text { Hypertensive/other } \\
\text { cardiovascular disease, \% }\end{array}$ & 37.8 & 25.4 & $<0.001$ \\
\hline Type 2 diabetes, \% & 14.5 & 10.0 & $<0.001$ \\
\hline Malignant cancer, \% & 6.6 & 3.8 & $<0.001$ \\
\hline Osteoporosis, \% & 3.4 & 1.3 & $<0.001$ \\
\hline Mood affective disorders, \% & 20.8 & 11.0 & $<0.001$ \\
\hline $\begin{array}{l}\text { Chronic obstructive } \\
\text { pulmonary disease, \% }\end{array}$ & 1.8 & 0.7 & $<0.001$ \\
\hline Chronic kidney disease, \% & 6.6 & 1.8 & $<0.001$ \\
\hline Liver disease, \% & 7.3 & 2.9 & \\
\hline
\end{tabular}

PoD, paediatric-onset disability.

and pharmacy (mean difference $=\mathrm{US} \$ 1793 ; \quad \mathrm{CR}=1.95$; 95\% CI=1.93 to 1.97 ) costs. The patient out-of-pocket costs (figure 1B) were higher for individuals with PoDs compared with individuals without PoDs for total (mean 

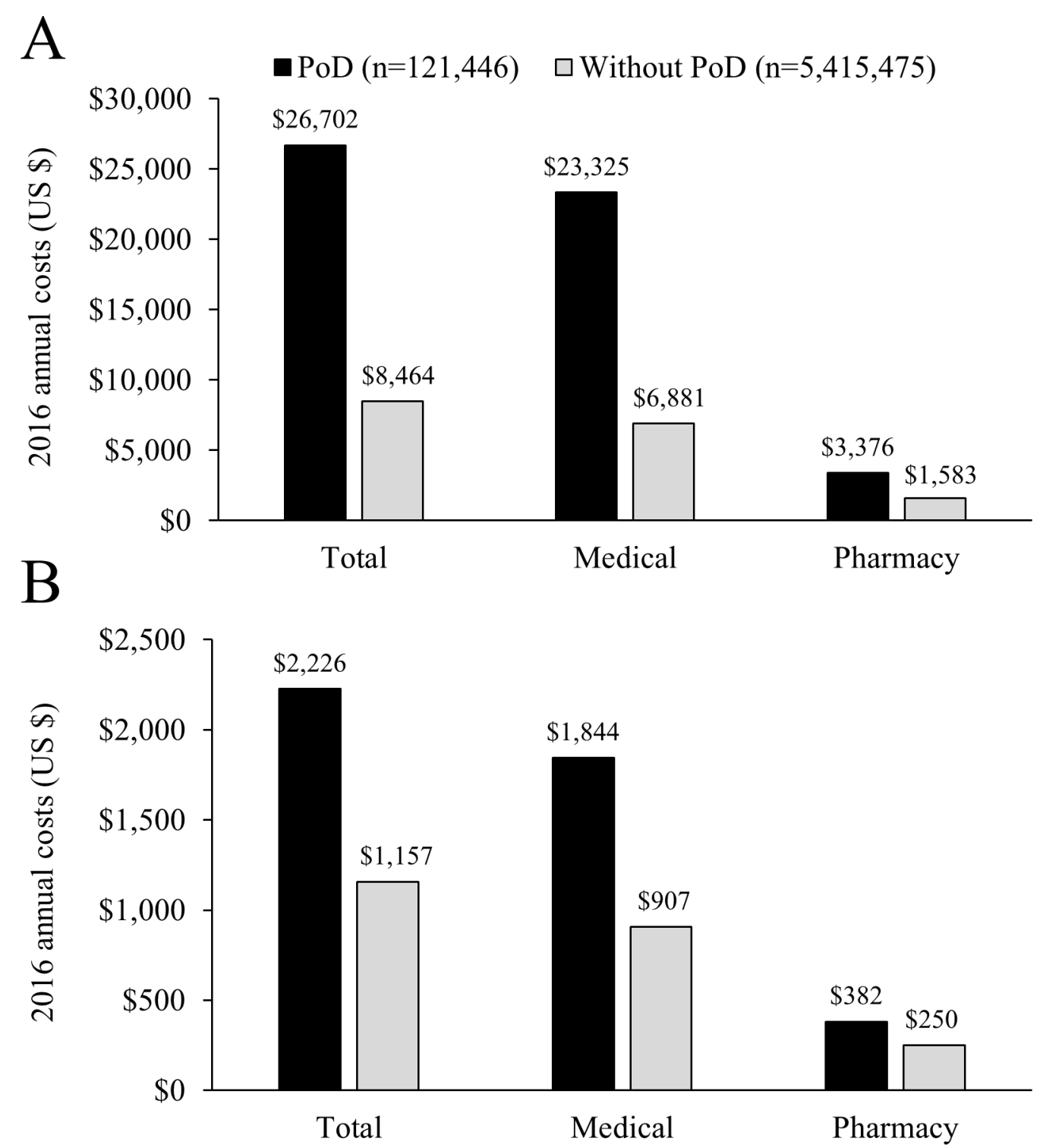

Figure 1 Annual mean all-cause total, medical and pharmacy standardised reimbursement costs $(A)$ and annual mean all-cause total, medical and pharmacy patient out-of-pocket costs (B) among adults (aged 18-64 years) with and without paediatric-onset disabilities (PoDs).

difference=US $\$ 1069 ; \mathrm{CR}=1.88 ; 95 \% \mathrm{CI}=1.86$ to 1.89$)$, medical (mean difference $=\mathrm{US} \$ 937 ; \quad \mathrm{CR}=1.96 ; 95 \%$ $\mathrm{CI}=1.95$ to 1.98 ) and pharmacy (mean difference $=\mathrm{US} \$ 132$; $\mathrm{CR}=1.39 ; 95 \% \mathrm{CI}=1.38$ to 1.40 ) costs.

Annual all-cause standardised reimbursement healthcare costs after adjusting for age, sex, education, insurance coverage and non-communicable diseases are presented in table 3. Individuals with PoDs had higher total $(\mathrm{CR}=2.32 ; 95 \% \mathrm{CI}=2.30$ to 2.34$)$, medical $(\mathrm{CR}=2.47$; $95 \% \mathrm{CI}=2.45$ to 2.49$)$ and pharmacy $(\mathrm{CR}=1.65 ; 95 \%$ $\mathrm{CI}=1.63$ to 1.66$)$ standardised reimbursement costs. All-cause patient out-of-pocket healthcare costs after adjusting for age, sex, education, insurance coverage and non-communicable diseases are presented in table 4. Individuals with PoDs had higher total (CR=1.65; 95\% $\mathrm{CI}=1.64$ to 1.66$)$, medical $(\mathrm{CR}=1.72 ; 95 \% \mathrm{CI}=1.71$ to 1.73$)$ and pharmacy $(\mathrm{CR}=1.30 ; 95 \% \mathrm{CI}=1.29$ to 1.31$)$ patient out-of-pocket costs. The sensitivity analysis revealed that ethnicity and household annual income did not affect the results $(\mathrm{n}=3545206)$ for total $(\mathrm{CR}=2.35 ; 95 \% \mathrm{CI}=2.33$ to 2.38$)$, medical $(\mathrm{CR}=2.48$; $95 \% \mathrm{CI}=2.45$ to 2.50$)$ and pharmacy $(\mathrm{CR}=1.79 ; 95 \% \mathrm{CI}=1.76$ to 1.81$)$ standardised reimbursement costs, or for total $(\mathrm{CR}=1.62 ; 95 \% \mathrm{CI}=1.61$ to 1.64$)$, medical $(\mathrm{CR}=1.68 ; 95 \% \mathrm{CI}=1.67$ to 1.70$)$ and pharmacy ( $\mathrm{CR}=1.34 ; 95 \% \mathrm{CI}=1.32$ to 1.35$)$ patient out-ofpocket costs.

Healthcare resource utilisation and costs by PoD category

Unadjusted healthcare resource utilisation for each PoD category is presented in online supplementary table 3. After adjusting for age, sex, education, insurance coverage and non-communicable diseases, all PoD categories had higher healthcare resource utilisation compared with individuals without PoDs (all $\mathrm{p}<0.001$ ), except for PoDs of the genital organs for inpatient visits $(\mathrm{p}=0.44)$ and PoDs of malformations of the eye, ear, face 
Table 3 Annual adjusted cost ratios (CR) for total, medical and pharmacy standardised reimbursement costs for year 2016 among adults (aged 18-64 years) with and without paediatric-onset disabilities (PoDs)

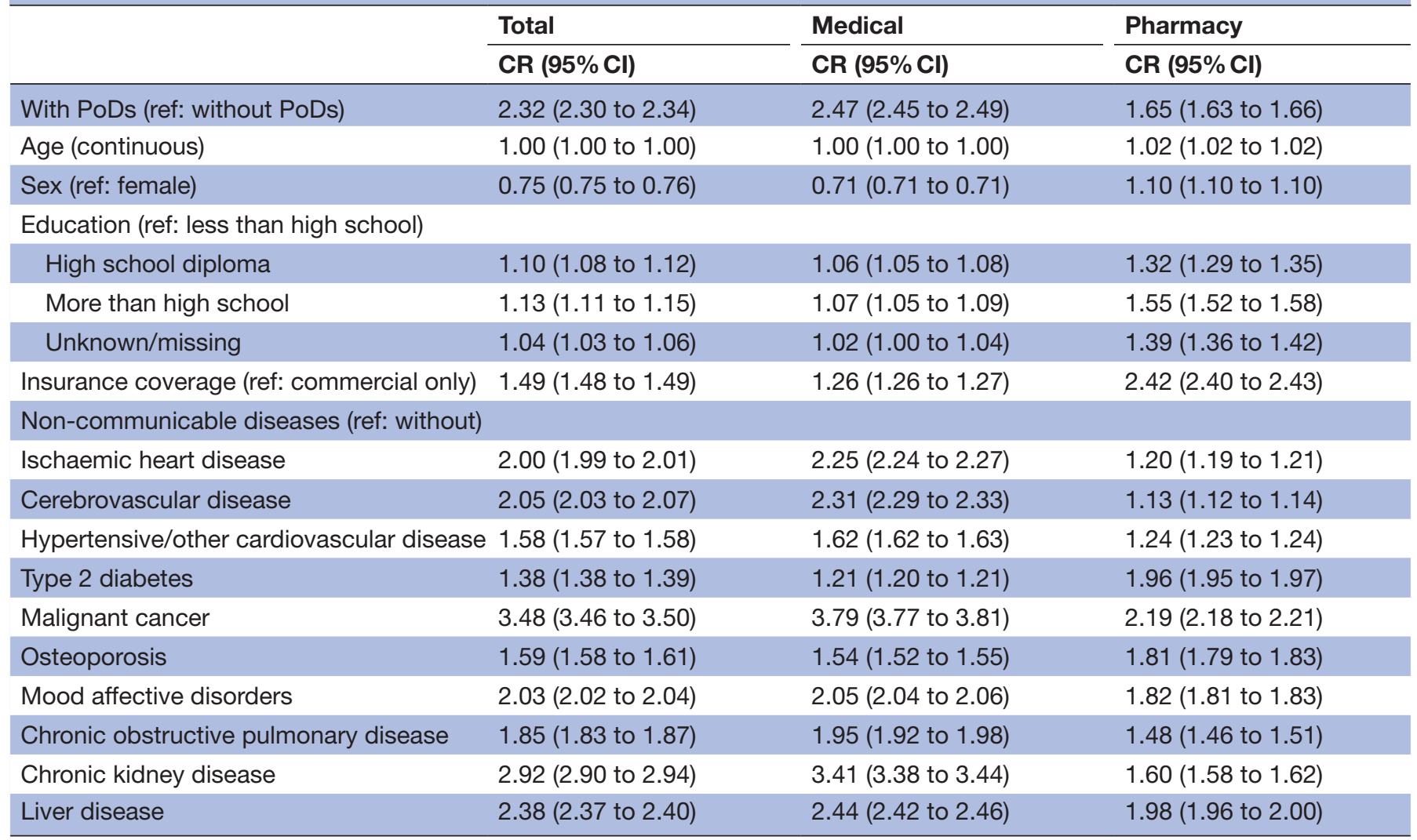

Generalised linear models with gamma distribution and log-link function were performed to estimate the $\mathrm{CR}$ and $95 \% \mathrm{Cl}$, which is the exponentiated form of the parameter estimate $n=5536921$.

and neck for emergency department visits $(\mathrm{p}=0.33)$. Unadjusted all-cause total healthcare costs for each PoD category are presented in figure 2. After adjusting for age, sex, education, insurance coverage and non-communicable diseases, all PoD categories had higher standardised reimbursement costs and patient out-of-pocket costs (all $\mathrm{p}<0.001)$.

Age by group interaction for healthcare costs for all adults with PoDs and by PoD category

There were significant age group (18-40years; 41-64years) by group (with PoDs; without PoDs) interactions for all individuals with PoDs and for each of the PoD categories for total standardised reimbursement and patient out-of-pocket costs (all $\mathrm{p}<0.001)$. After stratifying by young and middle-aged, all individuals with PoDs and each PoD category had higher CRs compared with individuals without PoDs, with the magnitude of the CR suggesting costs may be greater for young than middleaged individuals with PoDs (online supplementary file 4).

\section{DISCUSSION}

The principal finding of this investigation is that young and middle-aged privately insured adults with PoDs had higher healthcare resource utilisation across all medical services, and higher all-cause healthcare costs compared with young and middle-aged privately insured adults without PoDs. These findings were evident across all PoD categories when compared with individuals without PoDs. Moreover, the differences in costs were greater for younger than middle-aged adults with PoDs, and for each PoD category, compared with adults without PoDs. While previous studies examining adverse health complications among populations with PoDs have suggested earlier screening strategies for disease prevention and healthcare management, ${ }^{313}$ the findings from the current study could inform decision-making processes regarding private health benefit plan design and healthcare resource allocation for services and treatments by administrators and policymakers.

In the current study, we found substantial mean differences for all-cause healthcare costs and large unadjusted CRs for the standardised reimbursement and patient out-of-pocket costs between individuals with and without PoDs. After adjusting for sociodemographics, insurance coverage and the presence of several costly non-communicable diseases, total standardised reimbursement costs were still 2.3 times higher and patient out-of-pocket costs were 1.7 times higher for individuals with PoDs compared with individuals without PoDs. These results suggest a few things. First, costly non-communicable diseases only account for a small portion of the excess healthcare costs 
Table 4 Annual adjusted cost ratios (CR) for total, medical and pharmacy patient out-of-pocket costs for year 2016 among adults (18-64 years) with and without paediatric-onset disabilities (PoDs)

\begin{tabular}{|c|c|c|c|}
\hline & Total & Medical & Pharmacy \\
\hline & CR $(95 \% \mathrm{Cl})$ & CR $(95 \% \mathrm{Cl})$ & CR (95\% Cl) \\
\hline With PoDs (ref: without PoDs) & 1.65 (1.64 to 1.66$)$ & $1.72(1.71$ to 1.73$)$ & $1.30(1.29$ to 1.31$)$ \\
\hline Sex (ref: female) & 0.80 (0.80 to 0.81$)$ & 0.79 (0.79 to 0.79$)$ & 0.91 (0.91 to 0.91$)$ \\
\hline \multicolumn{4}{|l|}{ Education (ref: less than high school) } \\
\hline More than high school & 1.26 (1.24 to 1.28$)$ & 1.05 (1.04 to 1.07$)$ & $1.63(1.61$ to 1.66$)$ \\
\hline Unknown/missing & 1.17 (1.15 to 1.19$)$ & 1.04 (1.02 to 1.06$)$ & 1.55 (1.53 to 1.58$)$ \\
\hline Insurance coverage (ref: commercial only) & 0.81 (0.81 to 0.81$)$ & 0.70 (0.70 to 0.70$)$ & $1.31(1.30$ to 1.31$)$ \\
\hline \multicolumn{4}{|l|}{ Non-communicable diseases (ref: without) } \\
\hline Ischaemic heart disease & 1.54 (1.53 to 1.55$)$ & 1.64 (1.63 to 1.65$)$ & $1.22(1.21$ to 1.22$)$ \\
\hline Type 2 diabetes & 1.24 (1.24 to 1.24$)$ & 1.10 (1.10 to 1.10$)$ & $1.63(1.63$ to 1.64$)$ \\
\hline Malignant cancer & 1.78 (1.77 to 1.79$)$ & 1.93 (1.92 to 1.94$)$ & $1.16(1.15$ to 1.16$)$ \\
\hline Osteoporosis & 1.28 (1.27 to 1.30$)$ & 1.27 (1.26 to 1.29$)$ & 1.29 (1.28 to 1.30$)$ \\
\hline Mood affective disorders & 1.64 (1.63 to 1.64$)$ & 1.58 (1.58 to 1.59$)$ & 1.69 (1.68 to 1.69$)$ \\
\hline Chronic obstructive pulmonary disease & 1.45 (1.44 to 1.47$)$ & $1.53(1.51$ to 1.55$)$ & $1.23(1.22$ to 1.25$)$ \\
\hline Chronic kidney disease & 1.45 (1.44 to 1.46$)$ & 1.66 (1.65 to 1.67$)$ & $1.16(1.15$ to 1.16$)$ \\
\hline Liver disease & 1.61 (1.60 to 1.62$)$ & 1.74 (1.73 to 1.75$)$ & $1.14(1.13$ to 1.14$)$ \\
\hline
\end{tabular}

Generalised linear models with gamma distribution and log-link function were performed to estimate the $\mathrm{CR}$ and $95 \% \mathrm{Cl}$, which is the exponentiated form of the parameter estimate: $n=5536921$.

attributable to PoDs; however, there may be other costly diseases not examined in this study that are more prevalent among individuals with PoDs. Second, the findings that patient out-of-pocket costs were elevated for individuals with PoDs compared with individuals without PoDs, but to a lesser extent than for standardised reimbursement costs, suggests that health plans are accommodating some, but not all, of the medical needs by individuals with PoDs. This is supported by the higher prevalence of Medicare Advantage health plans among individuals with PoDs compared with individuals without PoDs (21.1\% vs 6.6\%). Furthermore, the CR for Medicare Advantage was higher compared with commercial alone for standardised reimbursement costs, but lower for patient out-of-pocket costs for the entire sample, suggesting greater cost reduction plans. Nevertheless, identifying and delineating other 'cost drivers' for populations with PoDs is needed.

The sample size for the group with PoDs was very large, which allowed us to examine healthcare resource utilisation and costs after stratifying by the type of $\mathrm{PoD}$, with sample sizes ranging from 5518 (PoDs with chromosomal abnormalities) to 33566 (PoDs of the musculoskeletal system). Our analyses suggest that all PoD categories have higher healthcare resource utilisation and costs compared with adults without PoDs. Moreover, there was a considerable range of excess healthcare utilisation and costs across PoD categories. In general, PoD categories with the highest healthcare resource utilisation and costs were PoDs of the urinary, circulatory, respiratory and digestive and nervous systems. While different types of PoDs should not be assumed to have the same set of health complications, as most have very specific aetiologies and comorbidities, there are overlapping risk factors across various types of PoDs that may impede healthful transition into and throughout adulthood, thus leading to similar health outcomes and healthcare needs. These factors may manifest as a direct consequence of the condition (eg, impaired executive functioning) or as an indirect consequence of a condition (eg, chronic pain, low societal integration). On the other hand, individuals with specific PoDs may be more susceptible to certain adverse health outcomes and reliantly on specific healthcare services. For example, children and adolescents with PoDs have higher prevalence of mental health disorders compared with the general population of children, ${ }^{30} 31$ but the prevalence is greater among children and adolescents with neurodevelopmental PoDs than nervous system PoDs. ${ }^{30}$ Future research is needed to parse out the PoD-specific factors contributing to excess healthcare utilisation and costs to lessen the healthcare economic burden attributable to various PoDs. 
A

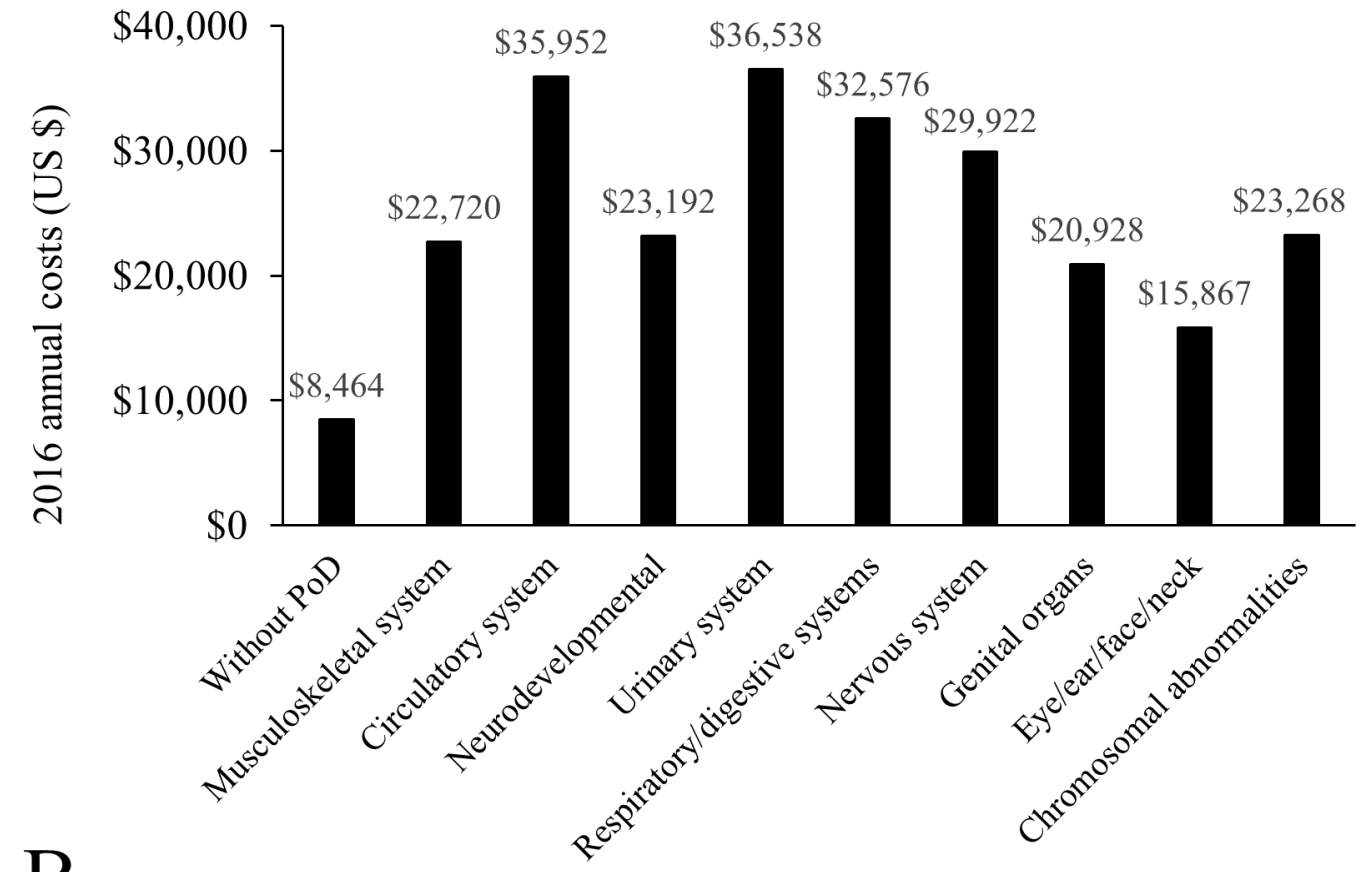

B

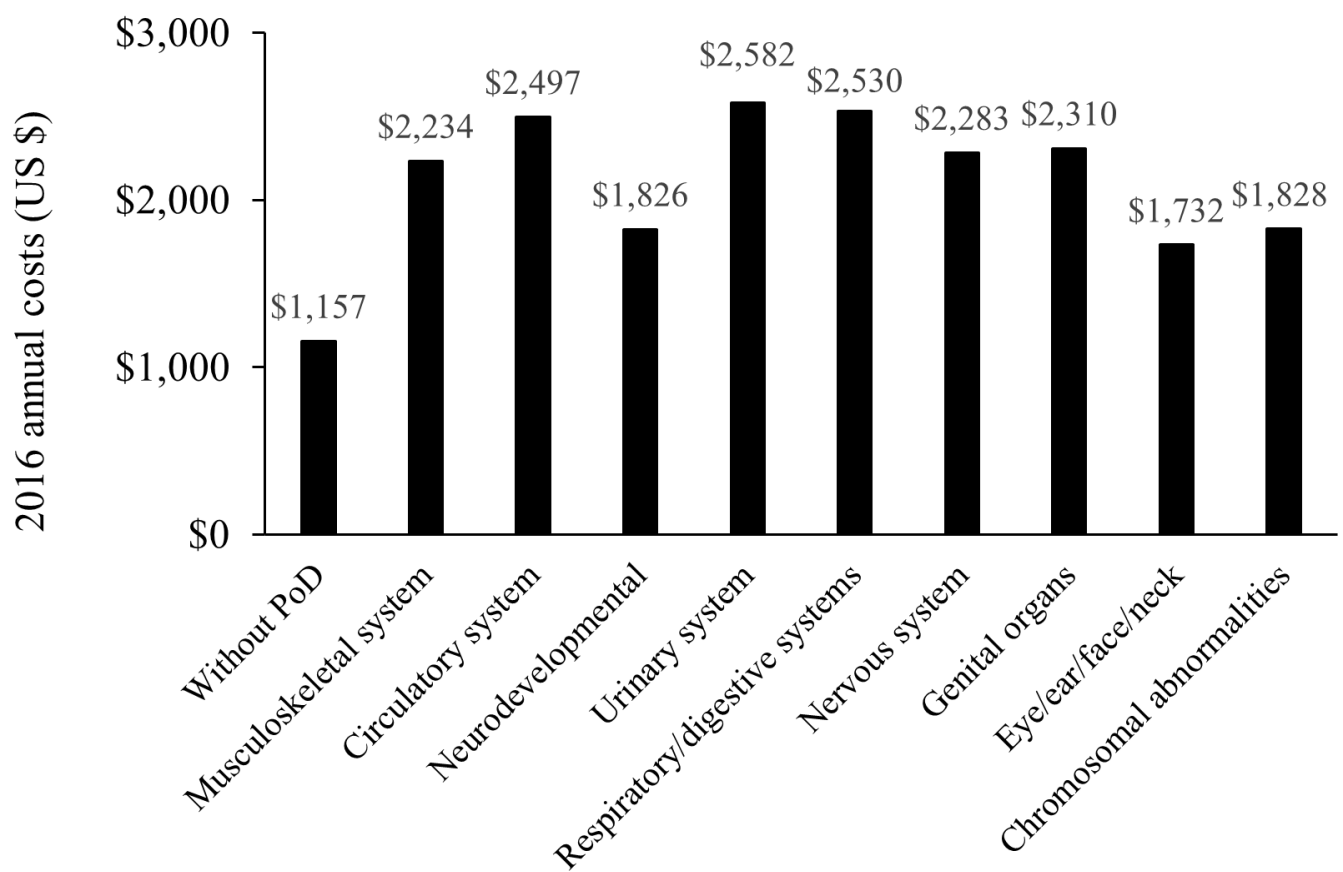

Figure 2 Annual mean all-cause total standardised reimbursement costs (A) and annual mean all-cause total patient out-ofpocket costs (B) among adults (aged 18-64 years) with and without paediatric-onset disabilities (PoDs), stratified by the type of PoD. Individuals may have more than one PoD and can be represented across multiple PoD categories. All PoD categories had higher costs compared with individuals without PoDs after adjusting for age, sex, education, insurance coverage and several non-communicable diseases (all $p<0.001$ ).

A major strength of the study included the large sample size for adults with PoDs. Gathering data on clinical populations is challenging and very little is known about health disparities among individuals with PoDs across the lifespan. Another major strength of this study is the comprehensive assessment of several costly non-communicable diseases which allowed for cost adjustment.
The limitations of the study must also be discussed. First, it is important to note that the findings from the current investigation are likely under-reporting the extent of healthcare resource utilisation and costs associated with US populations with PoDs. To be enrolled with a private health insurance plan, individuals must be able to afford the costs or be covered through their employer, 
their parents (up to 26years of age) or their spouse. Individuals with PoDs tend to have lower employment and marriage rates compared with the general population, ${ }^{33}$ which is likely to be more problematic with more medically complex forms of PoDs. Furthermore, individuals with more severe forms of PoDs are likely to be covered, or co-covered, by public health insurance due to medical circumstances. Therefore, our sample of adults with PoDs likely reflects a higher functioning and healthier segment of the US population with PoDs ${ }^{34}$; however, this is only speculation. Second, the present study excluded individuals that did not have service utilisation in 2016, which may have biassed results. However, these excluded individuals that had insurance coverage may be somewhat healthier since they did not require a medical encounter in 2016, thus potentially biassing results in the present study to be more conservative estimates. Third, data were from a single year and longer study periods and longitudinal research designs could provide more robust findings and implications for research, practice and policy. Fourth, we were only able to adjust for a limited set of covariates, and we are unable to rule out other potential confounding factors. Fifth, we used a single claim to identify PoDs and non-communicable diseases. Validation studies tend to show that two or more claims for a medical condition improves ability to identify individuals with that condition. ${ }^{35}{ }^{36}$ However, single claim-based algorithms using claims data have moderate-to-high positive predictive value $(\sim 80 \%)$ and high sensitivity $(99 \%)$ to detect PoDs, ${ }^{35}$ and moderate-to-high sensitivity (up to $99 \%$ ) and specificity (up to $87 \%$ ) to detect a variety of costly non-communicable diseases, ${ }^{37} 38$ although the accuracy of medical condition identification using claims data depends on the length of the study period ${ }^{39}$ and the condition examined. ${ }^{35} 373940$ Given the short study period of 12 months to extract data and the large and robust effect sizes, the selected methodology to identify associations is likely sufficient to provide evidence of health and economic disparities.

In conclusion, young and middle-aged adults with PoDs have higher healthcare resource utilisation and costs compared with young and middle-aged adults without PoDs. These higher costs come along with higher out-ofpocket burden, which can affect the individual's financial well-being, thus further contributing to health disparities. The elevated healthcare costs were evident even after adjusting for several costly non-communicable diseases that are more prevalent among populations with PoDs. Furthermore, each PoD category had higher healthcare resource utilisation and costs compared with individuals without PoDs. Future research is needed to identify specific cost drivers for the healthcare economic disparity for individuals with PoDs, and by the type of PoD, as well as year-to-year healthcare costs, which may provide insight into the long-term financial burden. Future research is also needed to develop algorithms and strategies for disease and cost prediction for these populations, which may significantly enhance preventive and personalised medicine, improve healthful ageing and reduce longterm costs. $^{41}$

Contributors DW conceived and designed the study, analysed the data and wrote the first draft of the manuscript. NK assisted in statistical analysis and interpretation. EAH, RAH and MDP assisted in interpretation of data. All authors approved the final manuscript for submission, and agree to be accountable for all aspects of the work.

Funding This work was supported by the University of Michigan Office of Health Equity and Inclusion Diversity Fund.

Competing interests None declared.

Patient consent for publication Not required.

Provenance and peer review Not commissioned; externally peer reviewed.

Data availability statement Data are available on reasonable request.

Open access This is an open access article distributed in accordance with the Creative Commons Attribution Non Commercial (CC BY-NC 4.0) license, which permits others to distribute, remix, adapt, build upon this work non-commercially, and license their derivative works on different terms, provided the original work is properly cited, appropriate credit is given, any changes made indicated, and the use is non-commercial. See: http://creativecommons.org/licenses/by-nc/4.0/.

\section{REFERENCES}

1. Verschuren $O$, Smorenburg ARP, Luiking $Y$, et al. Determinants of muscle preservation in individuals with cerebral palsy across the lifespan: a narrative review of the literature. J Cachexia Sarcopenia Muscle 2018.

2. Whitney DG, Hurvitz EA, Devlin MJ, et al. Age trajectories of musculoskeletal morbidities in adults with cerebral palsy. Bone 2018;114:285-91.

3. Whitney D, Hurvitz E, Ryan J, et al. Noncommunicable disease and multimorbidity in young adults with cerebral palsy. Clin Epidemiol 2018;10:511-9.

4. Charlson FJ, Baxter AJ, Dua T, et al. Chapter 3 Excess Mortality from Mental, Neurological, and Substance Use Disorders in the Global Burden of Disease Study 2010. In: Mental, Neurological, and Substance Use Disorders: Disease Control Priorities. Vol 4. Third Edition. Washington DC, 2016.

5. Hosking FJ, Carey IM, Shah SM, et al. Mortality among adults with intellectual disability in England: comparisons with the general population. Am J Public Health 2016;106:1483-90.

6. Pikora TJ, Bourke J, Bathgate K, et al. Health conditions and their impact among adolescents and young adults with Down syndrome. PLoS One 2014;9:e96868.

7. Sipes M, Matson JL, Belva B, et al. The relationship among side effects associated with anti-epileptic medications in those with intellectual disability. Res Dev Disabil 2011;32:1646-51.

8. Hermans H, Evenhuis HM. Factors associated with depression and anxiety in older adults with intellectual disabilities: results of the healthy ageing and intellectual disabilities study. Int J Geriatr Psychiatry 2013;28:691-9.

9. Colver A, Rapp M, Eisemann N, et al. Self-Reported quality of life of adolescents with cerebral palsy: a cross-sectional and longitudinal analysis. The Lancet 2015;385:705-16.

10. Groh WJ. Arrhythmias in the muscular dystrophies. Heart Rhythm 2012;9:1890-5.

11. Van Der Slot WMA, Nieuwenhuijsen C, VAN DEN BERG-EMONS RJG, et al. Chronic pain, fatigue, and depressive symptoms in adults with spastic bilateral cerebral palsy. Dev Med Child Neurol 2012;54:836-42.

12. Peterson MD, Kamdar N, Hurvitz EA. Age-Related trends in cardiometabolic disease among adults with cerebral palsy. Dev Med Child Neurol 2018.

13. Cremer N, Hurvitz EA, Peterson MD. Multimorbidity in middle-aged adults with cerebral palsy. Am J Med 2017;130:744.e9-744.e15.

14. Boyle CA, Boulet S, Schieve LA, et al. Trends in the prevalence of developmental disabilities in US children, 1997-2008. Pediatrics 2011;127:1034-42.

15. Brooks JC, Strauss DJ, Shavelle RM, et al. Recent trends in cerebral palsy survival. Part I: period and cohort effects. Dev Med Child Neurol 2014;56:1059-64.

16. Global Research on Developmental Disabilities Collaborators. Developmental disabilities among children younger than 5 years in 195 countries and territories, 1990-2016: a systematic analysis 
for the global burden of disease study 2016. Lancet Glob Health 2018;6:e1100-21.

17. Berry JG, Berry SD. Caring for patients with neurological impairment: conversations between a pediatrician and Geriatrician. JAMA Pediatr 2018.

18. Ahmedani BK, Hock RM. Health care access and treatment for children with co-morbid autism and psychiatric conditions. Soc Psychiatry Psychiatr Epidemiol 2012;47:1807-14.

19. Vohra R, Madhavan S, Sambamoorthi U, et al. Access to services, quality of care, and family impact for children with autism, other developmental disabilities, and other mental health conditions. Autism 2014;18:815-26.

20. Aisen ML, Kerkovich D, Mast J, et al. Cerebral palsy: clinical care and neurological rehabilitation. The Lancet Neurology 2011;10:844-52.

21. Quiñones AR, Markwardt S, Botoseneanu A. Multimorbidity combinations and disability in older adults. J Gerontol A Biol Sci Med Sci 2016;71:823-30.

22. Salive ME. Multimorbidity in older adults. Epidemiol Rev 2013;35:75-83.

23. Whiteford HA, Degenhardt L, Rehm J, et al. Global burden of disease attributable to mental and substance use disorders: findings from the global burden of disease study 2010. The Lancet 2013;382:1575-86.

24. Murray CJL, Vos T, Lozano R, et al. Disability-adjusted life years (DALYs) for 291 diseases and injuries in 21 regions, 1990-2010: a systematic analysis for the global burden of disease study 2010 . The Lancet 2012;380:2197-223

25. GBD 2016 Causes of Death Collaborators. Global, regional, and national age-sex specific mortality for 264 causes of death, 19802016: a systematic analysis for the global burden of disease study 2016. Lancet 2017;390:1151-210.

26. GBD 2017 DALYs and HALE Collaborators. Global, regional, and national disability-adjusted life-years (DALYs) for 359 diseases and injuries and healthy life expectancy (HALE) for 195 countries and territories, 1990-2017: a systematic analysis for the global burden of disease study 2017. Lancet 2018;392:1859-922.

27. Fekedulegn D, Andrew M, Violanti J, et al. Comparison of statistical approaches to evaluate factors associated with metabolic syndrome. J Clin Hypertens 2010;12:365-73.

28. Barber J, Thompson S. Multiple regression of cost data: use of generalised linear models. J Health Serv Res Policy 2004;9:197-204.
29. Diehr P, Yanez D, Ash A, et al. Methods for analyzing health care utilization and costs. Annu Rev Public Health 1999;20:125-44.

30. Whitney DG, Shapiro DN, Warschausky SA, et al. The contribution of neurologic disorders to the National prevalence of depression and anxiety problems among children and adolescents. Ann Epidemiol 2018.

31. Whitney DG, Warschausky SA, Peterson MD. Mental health disorders and physical risk factors in children with cerebral palsy: a crosssectional study. Dev Med Child Neurol 2018.

32. Whitney DG, Shapiro DN, Peterson MD, et al. Factors associated with depression and anxiety in children with intellectual disabilities. $J$ Intellect Disabil Res 2018.

33. Tumin D. Marriage trends among Americans with childhood-onset disabilities, 1997-2013. Disabil Health J 2016;9:713-8.

34. Whitney DG, Alford Al, Devlin MJ, et al. Adults with cerebral palsy have higher prevalence of fracture compared with adults without cerebral palsy independent of osteoporosis and cardiometabolic diseases. J Bone Miner Res 2019;34:1240-7.

35. Reeves S, Garcia E, Kleyn M, et al. Identifying sickle cell disease cases using administrative claims. Acad Pediatr 2014;14:S61-S67.

36. Kerr EA, McGlynn EA, Van Vorst KA, et al. Measuring antidepressant prescribing practice in a health care system using administrative data: implications for quality measurement and improvement. $J t$ Comm J Qual Improv 2000;26:203-16.

37. Doktorchik C, Patten S, Eastwood C, et al. Validation of a case definition for depression in administrative data against primary chart data as a reference standard. BMC Psychiatry 2019;19:9.

38. Kurdyak P, Lin E, Green D, et al. Validation of a population-based algorithm to detect chronic psychotic illness. Can J Psychiatry 2015;60:362-8.

39. Leslie WD, Lix LM, Yogendran MS. Validation of a case definition for osteoporosis disease surveillance. Osteoporos Int 2011;22:37-46.

40. Noyes K, Liu H, Lyness JM, et al. Medicare beneficiaries with depression: comparing diagnoses in claims data with the results of screening. PS 2011:62:1159-66.

41. Golubnitschaja O, Baban B, Boniolo G, et al. Medicine in the early twenty-first century: paradigm and anticipation - EPMA position paper 2016. Epma J 2016;7. 